\title{
A Survey on Comparative Study of Wireless Sensor Network Topologies
}

\author{
Jolly Soparia \\ Department of Information Technology, CSPIT, \\ Changa \\ Charotor University of Science and Technology, \\ Changa
}

\begin{abstract}
Wireless Sensor Networks have emerged as a new research Technology in the distributed computing environment and plays[1] as a major role in many applications nowadays. So based on the Topologies there can be varied applications. In this paper, a survey on various topologies of wireless sensor networks is carried out. The advantage and disadvantage have been also listed out. Also the comparison between the various topologies has been list out. This topology plays a very important role to support various applications. This paper describes that which topology is best for a particular application.
\end{abstract}

Keywords

Topology, Sensor Networks, PAN coordinator, Leader

\section{INTRODUCTION}

Generally, a Wireless Sensor Network can be defined as a collection of sensor nodes organized into a cooperatively network which can sense and control the environment enabling communication between persons or embedded computers and the surrounding environment [3]. Most of the conventional wireless systems, such as cellular telephone networks [4] and wireless local area networks (WLAN), make use of either Ring topology. Recently, however, a new wireless network topology, mesh topology, has been emerged with the increasing use of wireless sensor networks (WSN) in industrial applications. Sensor networks are capable of rendering local intelligence by sharing information about their local environment with nearby peers and further communicating this information to the central node where it can be processed and used to improve the performance of the network [4]. The need to supervise and measure various physical phenomena (e.g. temperature, fluid levels, vibration, strain, humidity, acidity, pumps, generators[11] to manufacturing lines, aviation, building maintenance and so forth) is common to many areas which includes home applications, health applications, forest fire detection, vehicle tracking, Industrial applications. But the cost of downloading, installing, maintaining, and upgrading makes wireless systems potentially attractive alternatives for general scenarios [2].

\section{WIRELESS SENSOR NETWORKS}

A Wireless Sensor Network is an gathering of millimeterscale, self-contained, micro-electro-mechanical [11] devices. These tiny devices are comprised of sensors, computational processing ability (i.e. CPU power), wireless receiver and transmitter technology and a power supply. In this network, a large number of sensor nodes usually span a physical geographic area. The interaction between sensor and controllers is a single hop i.e. it uses satellite or a centralized controller. In future, a WSN could be a collection of autonomous nodes or terminals that communicate with each other which forms a multi-hop radio network and maintaining

\author{
Nirav Bhatt \\ Department of Information Technology, CSPIT, \\ Changa \\ Charotor University of Science and Technology, \\ Changa
}

connectivity in a decentralized manner by forming an ad hoc network. Such WSN's could change their topology dynamically when connectivity among the nodes alters with time due to node mobility. But recent real-world deployment usually consists of stationary sensor nodes. WSN's are intelligent in comparison with traditional sensors, and some are designed to make use of in-network processing in which sensed data can be gathered in situ and metamorphosed to more abstract and aggregated high-level data before transmission. The combination of storage, processing power and wireless communication even depicts that data can be imbibed and dispersed using smart algorithms. Intuitively, a denser infrastructure will create a more effective sensor network. Thus, it provides higher accuracy and more energy is available for aggregation. If it is not properly handled, a denser network can also result into collisions during transmission of data that results into network congestion. This leads to increased latency and reduces efficiency in terms of energy consumption. One distinguishing feature of WSN's is the lack of strong boundaries between sensing, communication and computation. Unlike the Internet, in sensor networks every node is both a router and a data source [5]. The figure below shows the Wireless Sensor Network Overview:

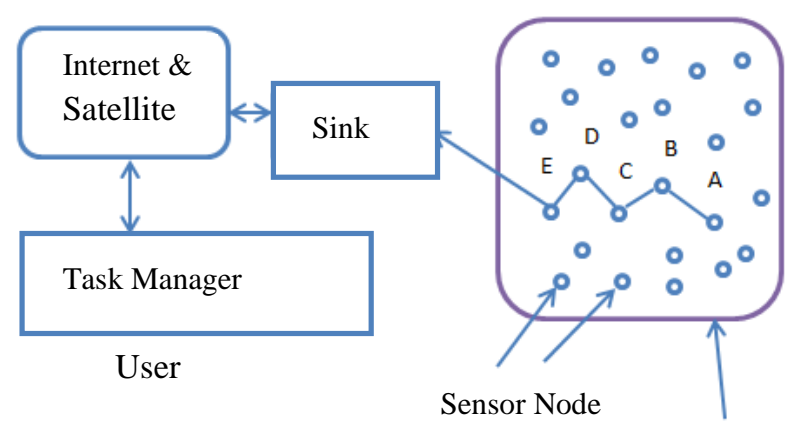

Sensor Field

Figure. 1 WSN Overview

\section{SENSOR NETWORK TOPOLOGIES 3.1 Star Topology}

In Star Topology, all sensor nodes are connected to a single node which can be called as a central node that works like PAN (Personnel Area Network) coordinator.

In this topology, all sensor networks are in direct communication range i.e. 30-100 meters to the central node. PAN coordinator functions to handle the decision making capabilities and routing. 


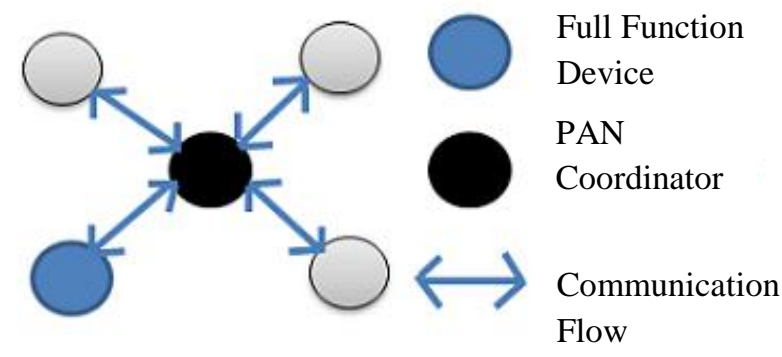

Figure 2. Star Topology

The end nodes do not forward the data or communication to each other rather they make use of central node as a communication point. If the node used for communication is collapsed, then it does not affect the entire network but only affects the particular node. If the central node is crashed, then whole sensor network is affected. A monitoring PAN monitors in medical field such as the blood pressure, glucose level, and gives the reading wirelessly and then transmits the data to the doctor performing rounds.

Therefore, Star Topology is well suited for such application. Battery resources of any node in the PAN coordinator will rapidly be ruined. Due to this, 802.15.4 standard recommended star topology for applications like games, toys as well as home automation.

\subsection{Cluster Tree Topology}

This is a special case of peer-to-peer topology in which most of the nodes are full-functional devices. Among all nodes, one node will be specified as the PAN coordinator due to which the network can be identified

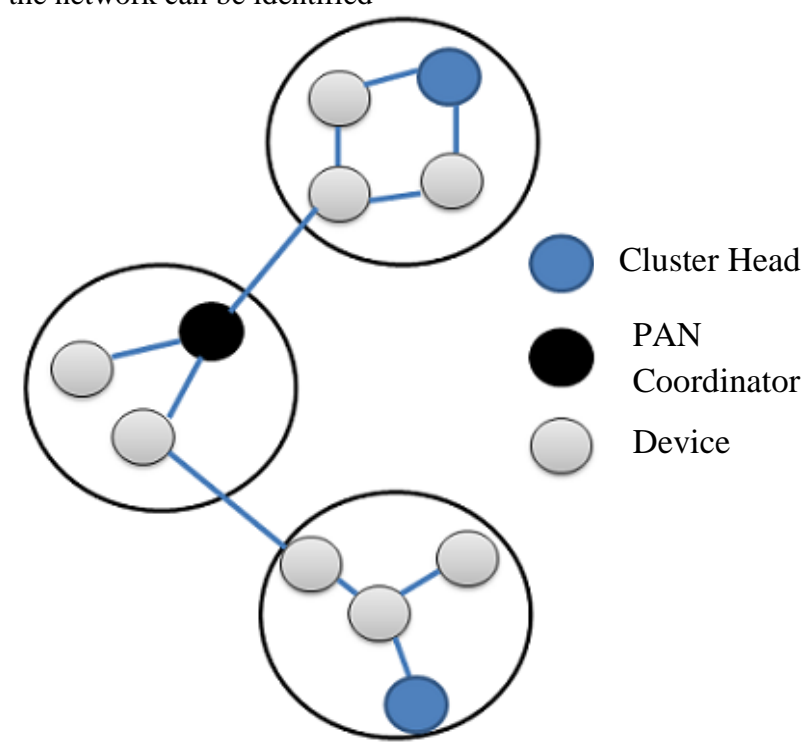

Figure 3. Cluster Tree Topology

The first cluster id made by PAN coordinator designating itself as a cluster Head has a cluster identifier value of zero. PAN coordinator sends the beacon. The device that receives the beacon can request to join the network. If the coordinator accepts the request, the requesting device is added as a child in its neighbor list. The newly joined device adds the Cluster Head as its parent in its neighboring list. For a Large-scale wireless network a mesh of multiple neighboring clusters can be formed. [1]

\subsection{Mesh Topology}

Mesh topology are basically multi-hoping system in which all sensor nodes can communicate with central node as well as with each other. The propagation of data through mesh allows a sensor network to extend, in theoretical the range is extended unlimited. Sensor network is highly fault-tolerance as each sensor nodes have multiple path back to the central node and other nodes. If any node causes failure, then the network automatically reconfigures itself around the failed node. The major issues are not related to power and latency but the important issue is to expand the network over large buildings. Hence, Mesh topology is appropriate for energy management that transmits temperature to a controller to determine the energy usage
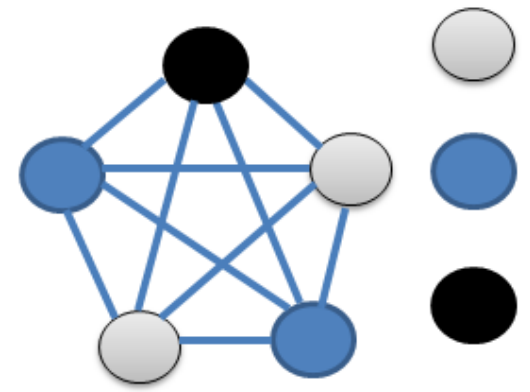

Reduced Function

Device

Full Function

Device

PAN

Coordinator

Figure 4. Mesh Topology

\subsection{Ring Topology}

As the name suggests, generally messages are passed in the ring. Only neighboring nodes are able to communicate with each other. If any node or link is broken during the communication, then it will affect the whole sensor network. Nowadays, ring topology is not preferred much in applications. One advantage of this sensor network topology is that it does not have any leader (central node).

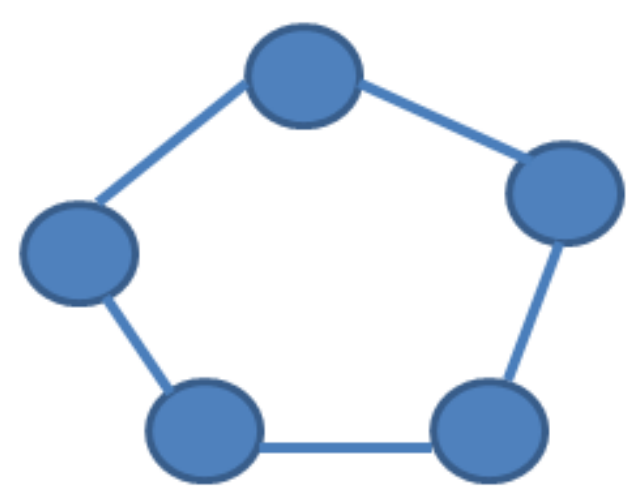

Figure 5. Ring Topology

\subsection{Star-Mesh Topology}

This topology has advantages of both star topology and mesh topology. Star topology is simple to implement and has low power consumption whereas self- healing nature of a mesh topology combines to implement Star-Mesh hybrid topology. The star-mesh hybrid organizes the nodes in star topology around mesh nodes, which they finally turn into a mesh 
network. The Mesh node provides flexibility to expand the range of the network and also fault tolerance. If one node fails in the network, then the radio link is send. The hybridization of Star-Mesh network offers the highest degree of sensor node mobility and flexibility for fast changes in the network population and the overall low power consumption. Hence for this reason, the star- mesh hybrid has proved to be logical choice for many implementation of wireless sensor networks. Applications such as industrial gas pad uses sensors in tanks to monitor fill levels.

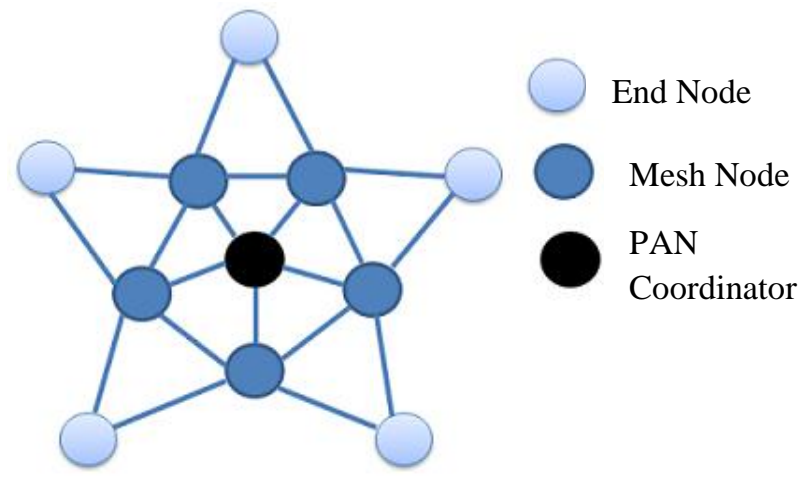

Figure 6. Star-Mesh Topology

\section{COMPARISON}

Table 1. Pros and Cons of various Topologies

\begin{tabular}{|c|c|c|}
\hline Topology & Advantages & Disadvantages \\
\hline Star topology & $\begin{array}{l}\text { Node Failure doesn't } \\
\text { affect the whole } \\
\text { network }\end{array}$ & $\begin{array}{l}\text { Coordinator failure } \\
\text { cause } \\
\text { network unable to } \\
\text { work }\end{array}$ \\
\hline Mesh topology & Highly-Fault Tolerant & $\begin{array}{l}\text { Mesh topology is } \\
\text { very complex }\end{array}$ \\
\hline $\begin{array}{ll}\text { Cluster } & \text { Tree } \\
\text { Topology } & \end{array}$ & $\begin{array}{l}\text { Node Failure doesn't } \\
\text { affect the whole } \\
\text { network }\end{array}$ & $\begin{array}{l}\text { Beacons must be } \\
\text { send to add more } \\
\text { nodes }\end{array}$ \\
\hline $\begin{array}{l}\text { Star-Mesh } \\
\text { Topology }\end{array}$ & $\begin{array}{l}\text { Highest Degree of } \\
\text { Mobility } \\
\begin{array}{l}\text { Flexibility to rapid } \\
\text { changes }\end{array}\end{array}$ & $\begin{array}{l}\text { It is very complex } \\
\text { and takes much } \\
\text { effort to handle than } \\
\text { any other Topology }\end{array}$ \\
\hline Ring Topology & $\begin{array}{l}\text { It is very simple } \\
\text { compared to other } \\
\text { Topologies }\end{array}$ & $\begin{array}{l}\text { Node Failure affects } \\
\text { whole network }\end{array}$ \\
\hline
\end{tabular}

The Table just summarizes us the advantage and disadvantage of the various Topologies.

Table 2. Various Topologies best suited application

\begin{tabular}{|l|l|l|}
\hline Topology & $\begin{array}{l}\text { Intercommunication } \\
\text { Between Nodes }\end{array}$ & \multicolumn{1}{c|}{$\begin{array}{c}\text { Application } \\
\text { (Best suited) }\end{array}$} \\
\hline Star topology & Not Allowed & $\begin{array}{l}\text { Games, Toys and } \\
\text { Home Automation }\end{array}$ \\
\hline Mesh topology & Allowed & Energy Management \\
\hline $\begin{array}{l}\text { Cluster Tree } \\
\text { Topology }\end{array}$ & Allowed & $\begin{array}{l}\text { Industrial data to be } \\
\text { stored or calculated }\end{array}$ \\
\hline
\end{tabular}

\begin{tabular}{|l|l|l|}
\hline $\begin{array}{l}\text { Star-Mesh } \\
\text { Topology }\end{array}$ & Allowed & $\begin{array}{l}\text { Industrial Gas Pad } \\
\text { application }\end{array}$ \\
\hline Ring Topology & $\begin{array}{l}\text { Only neighboring } \\
\text { nodes }\end{array}$ & $\begin{array}{l}\text { Not much in use in } \\
\text { any application }\end{array}$ \\
\hline
\end{tabular}

From this table one comes to know that every Topology is best suited for different applications. Hence based on the applications given one can use the best suitable topology. As for example one can say that if the application of games is given then the best suitable is the Star topology. Likewise if the Industrial based data is there to be managed then the Cluster Tree Topology is being used.

Hence different applications can behave differently or there may be a variation in output if the Topology is different for a particular application rather than best suited. Hence the developer must keep in mind the Topology and the application and then he must design the sensor network.

\section{CONCLUSION}

Wireless Sensor Networks have become an integral part of the life. The work presented here shows the comparative study of different topologies applied to wireless sensor network. Every Topology is needed or best suited to its particular application regardless of the Pros and Cons. Keeping all this topology in mind, hence one can find the best suited Topology for a particular application and hence can get the optimized resultant output.

In Future, Wireless Sensor network will be in use of day-today life like mobile phones. So as the applications based on Wireless Sensor Network are arising, it is seen that the topologies will play a major role. By analyzing the effect of this topologies, one can say the best Topology suited for an application and hence can get the expected result for particular applications.

There is a vast research area for Wireless Sensor Networks. Comparative study presented here can be extended to provide comparison with different factors for wireless sensor network apart from topology. So the Future of Wireless Sensor Network is going to be much brightening by having many more applications of it in many fields.

\section{REFERENCES}

[1] S. G. Shiva Prasad Yadav 1, Dr. A. Chitra 2, Wireless Sensor Networks - Architectures, Protocols,Simulators and Applications: a Survey, 1- Research Scholar, P. S. G. College of Technology, Coimbatore, 2- Professor, Department of Computer Science, P. S. G. College of Technology, Coimbatore

[2] I.F. Akyildiz, W. Su*, Y. Sankarasubramaniam, E. Cayirci, Wireless sensor networks: a survey, Computer Networks 38 (2002) 393-422.

[3] Verdone R, Dardari D, Mazzini G and Conti A ,Wireless Sensor and Actuator Networks - Technologies, Analysis and Design, London,UK, Elsevier, 2008

[4] Wireless Mesh Networks - Reliability and Flexibility

[5] Philip Levis $\dagger$, David Gaył, and David Culler†, Active Sensor Networks, $\uparrow$ EECS Department University of California, Berkeley, CA 94720, tIntel Research Berkeley 2150 Shattuck, Avenue.

[6] MeshScapeTM Technical Overview

[7] A. Koubaa, M. Alves, E. Tovar,IEEE 802.15.4 for Wireless Sensor Networks: A Technical Overview, IPP- 
HURRAY Technical Report, HURRAY-TR-050702, July 2005.

[8] KR KRISHNAIAH , Wireless Network Topologies

[9] Vikram Dhiman, INTERNATIONAL JOURNAL FOR ADVANCE RESEARCH IN ENGINEERING AND TECHNOLOGY,WINGSTO YOUR HOUGHTS.....,BIO Inspired Hybrid Routing Protocol for Wireless Sensor Networks, M.Tech ,NIT Jalandhar,Punjab, Pin no.14400, er.vikramdhiman@gmail.com.
[10] Eiko Yoneki, Jean Beacon, A survey of Wireless Sensor Network technologies: research trends and middleware's role, September 2005.

[11] Eiko Yoneki, Jean Beacon, A survey of Wireless Sensor Network technologies:research trends and middleware's role, powerpoint presentation. 